\title{
El método ODIT (Observación, Desarrollo e Ingeniería Turística) y su uso para la planificación estratégica y el desarrollo de los destinos turísticos
}

\section{Thiago Duarte Pimentel}

thiagodpimentel@gmail.com; Doctor en Ciencias Sociales/UFJF. Máster en Administración/UFMG. Licenciado en Turismo/UFMG. Profesor e investigador de tiempo completo en UFJF, postgrado en Ciencias Sociales y licenciaturas de Turismo y Ciencias Humanas. Miembro de la Asociación Internacional de Peritos Científicos en Turismo/AIEST. Visiting scholar en ASU/USA, UAS/Mx, La Habana/Cu, UTE/Ecu. Editor Jefe de Anais Brasileiros de Estudos Turísticos/ABET y Revista Latinoamericana de Turismologia/RLAT. Director de la red de investigación Conocimiento, Organización y Turismo/COGITO y del Observatorio Económico y Social de Turismo/OEST. Vice presidente del Consejo Municipal de Turismo/COMTUR, Juiz de Fora/MG. Brasil.

Recibido: 23 de marzo, 2015 • Aceptado: 11 de setiembre, 2015 • Corregido: 19 de octubre, 2015

\section{RESUMEN}

En el marco de la reciente política brasileña de popularización de la ciencia (scientific alphabetization), este artículo tiene como objetivo presentar y discutir, de manera esquemática, el método de planificación y gestión turística titulado ODIT - Observación, Desarrollo e Ingeniería Turísti$c a$. Su concepción busca integrar simultáneamente las premisas de sustentabilidad, participación democrática y autogestión con la utilización de procesos y técnicas formales con vistas a alcanzar eficacia técnica y profesionalizar la acción. Para ejecutarlo se hace uso de cuatro dimensiones: económica, social, ética y ambiental, que en suma otorgan una visión general de la visita in situ. Cada una estas dimensiones tiene un rol de indicadores para guiar la recopilación, la sistematización y el análisis de datos. Sin embargo, para tornar más preciso el análisis, la metodología ODIT divide la información en dos niveles: el nivel del territorio y el nivel de las empresas, con el fin de delimitar las escalas de análisis y de acción. En suma, este método se presenta como una de las formas más completas y bien elaboradas de planeación y gestión de los destinos turísticos.

Palabras clave: Método de Planificación Turística. Destino Turístico. Ingeniería Turística. Experiencia francesa.

Duarte Pimentel, T. (2016). El método ODIT (Observación, Desarrollo e Ingeniería Turística) y su uso para la planificación estratégica y el desarrollo de los destinos turísticos. Revista Espiga, Vol XV, (31), 45-57. 


\title{
ABSTRACT
}

The ODIT method (Observation, Development and Touristic Engineering) $y$ its use for the strategic planning and development of tourist destinations

\begin{abstract}
In the context of Brazil's recent policy of science popularizing (scientific alphabetization), this paper presents and discusses, in a schematic way, the method of tourism planning and management called ODIT - Observation, Development and Tourist Engineering. Its design seeks to simultaneously integrate the premises of sustainability, democratic participation and self-management with the use formal techniques and processes in order to achieve technical efficiency and professionalize the tourist destination management. To carry out this method, four dimensions - economic, social, ethical and environmental - work together to give an overview of the place analyzed. Each of these dimensions has its own set of indicators, which serves to both guide the data collection and systematize analysis. Yet, to get more control and precision, the ODIT method divides the information into two levels: the territory and the enterprise, in order to define the scale of analysis and action. In short, this method represents one the most complete and elaborate planning and management tools of tourist destinations.
\end{abstract}

Key words: Method of Tourism Planning. Tourist Destination. Tourist engineering. French experience.

\section{Introducción}

Motivado por la reciente política brasileña (CNPq / MCTI) de popularización de la ciencia (scientific alphabetization), este artículo tiene como objetivo presentar y discutir, de manera esquemática, el método de planificación y gestión turística titulado ODIT - Observación, Desarrollo e Ingeniería Turística, formulado por la Agencia Francesa de Ingeniería Turística /AFIT, particularmente creada para la planificación y gestión del turismo. Además, él proporciona un conjunto de técnicas y procedimientos sistemáticos capaces de guiar el proceso de planificación de una manera profesional y efectiva.

Específicamente se pretende: (1) presentar la metodología, específica del turismo, ODIT / Observación, Desarrollo e Ingeniería Turística, (2) explicar su funcionamiento a través de sus fases y principales características, dimensiones y escalas; y, finalmente, (3) ilustrar el proceso de desarrollo de indicadores de turismo, el cual está basado en la metodología ODIT. Estos indicadores son útiles para la gestión de destinos turísticos.
A pesar de las discusiones acerca de los datos históricos del inicio del turismo en el mundo dilema que se entrelaza con la competencia sobre la definición misma de lo que es el turismo - hay, de hecho, un consenso de que la planificación y la organización en esta temática son recientes, asumiendo como punto de referencia la institución de esta nueva fase en el último cuarto del siglo XIX (Costa, 2001), pero cuya intensificación y uso masivo solo se producen en la segunda mitad del siglo XX. A partir de ahí, con el crecimiento del turismo, los condicionantes de su desarrollo empezaron a ser cada vez más importantes para la realización de la actividad turística.

Pero, a medida que se intensifica, se aumenta su complejidad y la dependencia de los nuevos factores. El turismo ha cambiado la dinámica social y los espacios productivos donde se inserta, por lo que es imprescindible intervenir en la actividad de control de sus efectos perjudiciales y maximizar sus efectos positivos. Desde entonces, varios modelos emergieron para entender el turismo - modelos que también pasaron a servir como referencia para la intervención racional (a 
través de la planeación) en esta actividad - (Lohmann y Panosso Netto, 2008).

Según Acerenza (2003), uno de los primeros intentos de la gestión del turismo se llevó a cabo en 1948, en la región turística conocida como la costa de Aquitania, en el suroeste de Francia. En ese momento, el entendimiento del tema del turismo se relacionaba con un conjunto de adecuaciones en la infraestructura demandada por el espacio físico de las nuevas actividades públicas. Con el tiempo, se celebraron otras iniciativas en diversos destinos turísticos de todo el mundo y, como estaban aprendiendo de las experiencias anteriores, las mejoras cualitativas estaban siendo asimiladas por una nueva dirección y planificación del turismo.

El proceso de planificación históricamente ha sido tratado como una tarea administrativa. Sin embargo, a menudo, en el turismo este proceso se confunde metonímicamente con la propia gestión en su conjunto. Esta comprensión sintética de la gestión del turismo en términos de una sola actividad, la planificación, se ha hecho de manera constante y puede ser verificada a través del énfasis, a veces excesivo, que se tiene sobre las propuestas y los métodos de planificación (Stifitel, 1985).

Estos métodos colaboraron en la gestión de destinos, ya que permitieron planificar la actividad mediante la visualización del sistema turístico en su conjunto. A su vez, crearon medios para intervenir y controlar el flujo de turistas, adaptar la infraestructura, calificar el destino turístico, entre otros factores. Por otra parte, en la mayoría de los casos, se limitaron a aplicar los conocimientos de otras áreas - particularmente, la administración y la economía - en el turismo, sin desarrollar a partir de sus propios sistemas turísticos, técnicas, procedimientos y sistemas de información específicos para la gestión de los destinos.

Recientemente, entre finales de 1990 y principios del 2000, una innovadora iniciativa emprendida por el gobierno francés convocó a un grupo de expertos en planificación turística, y elaboró, a partir de una serie de estudios preliminares, un documento síntesis titulado Conducir el Turismo Sostenible en Territorios y Empresas: una guía de "saber hacer". Este documento elaborado por la Agencia Francesa de Ingeniería Turística (actualmente Agencia de Desarrollo Turístico de Francia/Atout France), pasó a servir como referencia metodológica oficial para la planificación turística territorial por varios organismos públicos franceses, como el Departamento de Estado de Turismo y el Ministerio de Planificación Territorial y Medio Ambiente.

Para llevar a cabo las operaciones necesarias de planificación, gestión y desarrollo turístico francés, la agencia Atout France estableció un triple objetivo: a) la promoción del turismo en Francia; b) la realización de las operaciones de planificación del turismo; c) la aplicación de una política de calidad y de competitividad de las empresas. Para lograr estos objetivos, Atout France necesitó la integración de dos organismos: 1) la ODIT Francia, inicialmente una asociación de interés público especializada en la planificación del turismo que se ocupaba de la estructura del turismo específicamente con las comunidades locales y los operadores privados; y 2) la Maison de la France, agrupación de interés económico a cargo de la promoción del turismo en Francia.

A medida que el Ministerio de Turismo de Brasil ha lanzado recientemente un conjunto de módulos de apoyo para ayudar a los profesionales del turismo para desarrollar la actividad más homogénea en el país, el mismo recurso de institucionalización de métodos de planeación que ya se utilizaba en otros países - fue puesto en práctica. Así, considerando las contribuciones múltiples del método ODIT es que se plantea la presentación y discusión de ese método como un caso ejemplar (Vandenberghe \& Boschi, 2013).

Básicamente, estos instrumentos se destinan a "normalizar" el desarrollo del turismo interno, orientando las acciones y las formas en que se llevarán a cabo en una dirección común. Pero, la diferencia entre los módulos de funcionamiento Embratur (EMBRATUR, 2007) y el ODIT es que este tiene algunas características 
distintivas específicas que pueden contribuir de forma decisiva a la planificación y a la gestión de los destinos turísticos de una manera más eficaz y profesional.

En general, hay dos características principales. La primera, en cuanto a una postura filosófica y teórica, donde se tiene el supuesto de la creación de una dinámica para ejercer el proceso de planificación que abarca la participación colectiva y la sostenibilidad. Para eso se utiliza una organización mediadora (preferiblemente externa al destino turístico en cuestión), que coordinará el proceso de planificación, lo que garantiza una mayor transparencia y equidad.

La segunda se refiere al marco metodológico con el conjunto de indicadores desarrollados específicamente para el turismo, cuyo objetivo es guiar la búsqueda de información sobre el estado actual del turismo local y ofrecer una mayor apropiación, precisión, profundidad y detalle a la propuesta.

En este sentido se justifica un mayor contacto y conocimiento de la propuesta metodológica francesa, la cual puede servir de estímulo para repensar las metodologías utilizadas al nivel nacional y, en su caso, su mejora y profundización.

\section{El método francés ODIT: Observación, Desarrollo e Ingeniería Turística}

El Turismo en Francia pasó por un período de rápido crecimiento cuantitativo entre finales de 1950 y finales de 1980. Sin embargo, más recientemente la industria del turismo francés busca un desarrollo cualitativo para adaptarse al crecimiento de la oferta global. El turismo en este país, a pesar de ser una actividad joven, ella ya adquirió un grado de madurez y, poco a poco, comienza a concentrarse en la aplicación empírica de un turismo sostenible en los territorios nacionales que muestran la disposición de aplicar este tipo de turismo y además otorgan la organización necesaria para lograrlo.

El turismo sostenible tiene como objetivo proporcionar estancias de calidad a los turistas mientras se busca mejorar la vida de las personas. También se interesa directamente por los impactos de las actividades turísticas en el desarrollo del territorio y por garantizar beneficios equitativos para las poblaciones locales. En términos de producción, el turismo sostenible aboga principalmente por desarrollar prácticas que permitan a las empresas turísticas maximizar la creación de riqueza, preservando y optimizando el capital ambiental y humano del territorio, la durabilidad necesaria y los atractivos del destino.

Sin embargo, para que el turismo se desarrolle en los territorios de forma sostenible, tal como propone el método ODIT, un número de factores deben ser tomados en consideración. En primer lugar, se necesita estar consciente de la relación entre el territorio y las empresas ahí ubicadas. Por lo tanto, es necesario observar las prácticas y estrategias que los actores ejercen en la actividad turística, tanto en escala empresarial como en la escala territorial.

En segundo lugar, se debe construir un plan de acción como resultado del proceso de planificación del turismo sostenible en el territorio, de una forma propia y precisa, llevado a cabo sistemática y profesionalmente. El proceso de planificación consiste en la organización de las acciones necesarias para alcanzar los objetivos previamente establecidos. Además, es el dispositivo de gestión más importante, pues funciona como elemento de orientación de la acción (plan) y también como herramienta de corrección (evaluación). Por lo tanto, para guiar el desarrollo de un plan de turismo sostenible de conformidad con las necesidades y deseos locales, es necesario el uso de la información recogida con anticipación desde el territorio y las organizaciones presentes en él.

Por último, se debe tomar en cuenta la diversidad de actores que participan en el turismo, de manera que su efecto en el entorno sea lo más beneficioso para el desarrollo del turismo sostenible. Esto es necesario pues el turismo no es una actividad aislada en el territorio, al contrario, ejerce y está influenciado por el entorno en el 
que opera, lo que podría afectar de manera importante las posibilidades de desarrollo turístico sostenible. Por lo tanto, para responder a múltiples demandas locales hay que tener en cuenta la diversidad de actores.

Sin embargo, es muy difícil y poco probable que los actores, debido a su diversidad, puedan articularse de manera democrática y equitativa para garantizar el derecho recíproco de expresar sus ideas y reivindicar sus intereses. Por lo tanto, es necesario que alguna entidad (por ejemplo, una firma consultora, una agencia estatal, un centro de investigación o una universidad), siempre que sea posible externa al territorio, ocupe el rol de mediador en este proceso.

Ese proceso de mediación (también conocido, en la terminología francesa, como "animación" de los actores) les lleva a reflexionar colectivamente sobre sus prácticas de turismo y valorar la diversidad mediante la organización de dispositivos favorables de su expresión, la creatividad y la validación colectiva de las directrices necesarias para lograr el turismo sostenible.

En resumen, el método ODIT tiene en cuenta distintas premisas - la sostenibilidad local, la participación colectiva, la transparencia- las cuales son reunidas de un modo muy particular, y además son puestas en marcha a través de una dinámica sistemática y profesional. Para dedicarse con más detalle a las cuestiones relacionadas con la experiencia francesa de la planificación y gestión del turismo, se tratará en este texto de la lógica del funcionamiento del método ODIT, desde un punto de vista teórico, pero validado empíricamente por una serie de trabajos aplicados en otras ocasiones (véase Pimentel, 2010, 2011, 2012, 2013).

\section{Lógica del Funcionamiento de ODIT}

Cada modelo de planificación del turismo presenta una secuencia de fases que se deben seguir para el logro de los efectos esperados. Recopilar datos, interpretarlos, diagnosticar problemas y señalar las intervenciones, son pasos que conducen a la formulación de un plan formal, que luego puede aplicarse o no, en dependencia del contexto. En el caso de la metodología ODIT, la secuencia de las fases ocurre de la siguiente forma: 1) el retrato del lugar, 2) el diagnóstico, 3) la definición de los ejes estratégicos, y 4) el plan de acción. (Fig. 1)

\section{Fase 1: El Retrato del Lugar}

La primera etapa de la aplicación del método ODIT es el Retrato del Lugar. Es decir, la comprensión de la relación entre la actividad turística y la ubicación en el entorno en que ella opera. Su objetivo es identificar con la mayor precisión y detalle posible la diversidad de actores - públicos y privados -, sus prácticas (acciones, hábitos, creencias y tradiciones) e intereses, así como las dinámicas existentes en el territorio (¿Cómo suceden las cosas en ese lugar? ¿Quién hace qué tipo de cosa? ¿Cómo se hacen? ¿Con qué frecuencia?)

Lo importante en esta etapa es analizar la diversidad de actores individuales (residentes, turistas, trabajadores, entre otros.) y colectivos (asociaciones, organizaciones, empresas, entre otros.) y las prácticas que se asocian con ellos. Más que eso, es necesario involucrarlos en el compromiso de construir colectivamente un conjunto de indicadores para la dirección del turismo sostenible en el territorio.

Sin embargo, debido a la diversidad de los actores, hay que tener en cuenta que ni siempre se puede comparar diferentes datos. Por ejemplo, la opinión de un habitante de la ciudad es una realidad diferente de la opinión institucional de la municipalidad o de un actor colectivo (por ejemplo, una empresa) ${ }^{1}$. Esta se refiere a un grupo de

1. Tradicionalmente hay el reconocimiento en la sociología de que la realidad social puede ser estudiada a partir de los niveles micro, meso y macro social. Para una adecuada y sintética explicación sobre los distintos niveles de la realidad social, más allá de estos, véase Brante, T. (2001) Consequências do realismo na construção de teoria sociológica. Sociología, set. 2001, nº.36, p.9-38. 


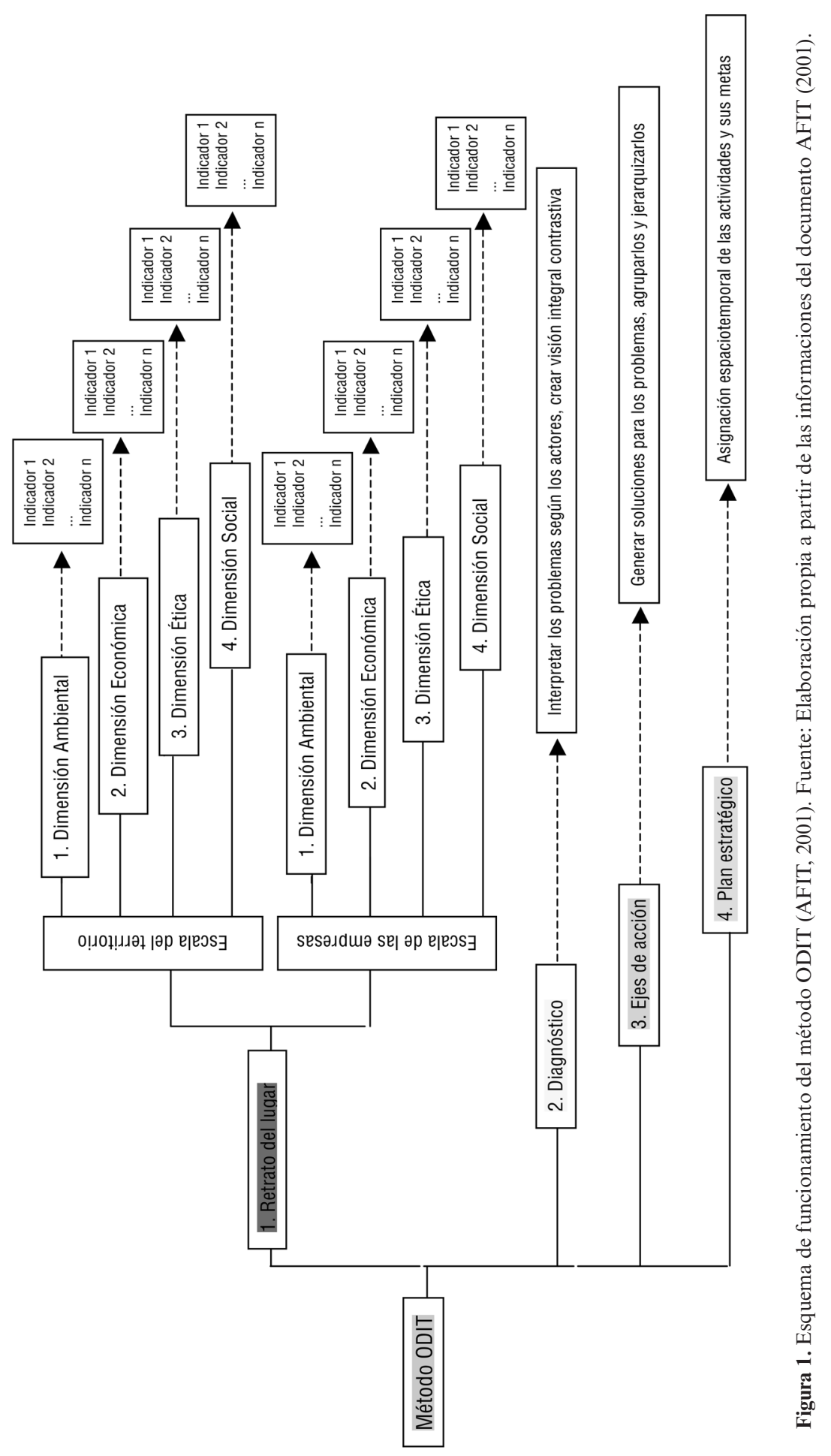


personas. Por lo tanto, la comparación de la opinión de un individuo o de una organización puede llevar a mal entendidos en la interpretación de los datos y, por supuesto, hay que considerar parámetros metodológicos seguros para su análisis.

Para evitar este tipo de situación, la cual puede influir considerablemente en el diagnóstico y, por lo tanto, en el resultado global del plan de turismo, el método ODIT sugiere dividir la recolección de los datos de la realidad y su análisis en dos niveles: el del territorio y el de la empresa, con el fin de delimitar focos de análisis y de acción distinta y no solapante.

En el primer nivel -el análisis territorial- el objetivo es identificar las cuestiones relacionadas con el territorio y el turismo por los actores. Por lo tanto, la diversidad de lógica y racionalidades de los actores es la guía de esa fase. En el segundo nivel -el análisis de las organizaciones, con énfasis en aquellas turísticas- el objetivo es identificar los tipos de prácticas y sus métodos de evaluación de la sostenibilidad, e identificar posibles mejoras. Este análisis permite prefigurar el modo de organización para facilitar la participación.

En lo que respecta al territorio, es preciso profundizar su dinámica con el turismo, la economía, la calidad de vida, entre otros. Hay que tener un mayor enfoque en el tema del turismo, buscando, por ejemplo, observar la interrelación entre los visitantes y lugareños, quienes toman ventaja de la oportunidad de desarrollar la ciudad turística económicamente y los impactos de las acciones entre ellos. Saber disfrutar de esta actividad y sus beneficios requiere una planificación, de ser posible dentro de la lógica del turismo sostenible, de manera que todos se beneficien.

Dentro de las empresas se debe analizar e identificar los puntos clave relacionados con sus procesos operativos y el turismo. Por ejemplo, cómo se desarrolla el turismo en la localidad, cuál es su progreso, se está llevando a cabo de una manera sostenible, qué se necesita mejorar y cómo hacerlo. Así se desarrollará el turismo con más facilidad, con mejor calidad y con la participación de todos los interesados.
Estas dos etapas culminan en una serie de preguntas clave para la comprensión de las dinámicas locales de turismo, donde:

- $\quad$ se identificará claramente lo que gobierna el comportamiento de los actores en el territorio y el turismo;

- $\quad$ se identificará y reunirá a la diversidad de las representaciones en el territorio y caracterizará las prácticas de turismo que dependen de ella;

- se tendrá en cuenta las formas de llevar a cabo las prácticas turísticas de las organizaciones;

- se conocerá las expectativas y prioridades de las organizaciones frente a las políticas territoriales y a las estructuras de supervisión.

Para hacerlo más claro, estos dos niveles se pueden considerar como las células humanas observadas a través de un microscopio: al nivel macro, donde se analizarían las células para ver cómo se relacionan entre sí y al formar el tejido tendríamos el nivel agregado del territorio. El nivel micro, es decir, dentro de las células, se podría comparar con la forma de producción de operaciones para su manutención. Así, en el caso del turismo, en el primer nivel, el macro, ocurre la recopilación de datos orientados a las cuestiones de territorio en su conjunto. Es decir, la suma de las unidades económicas, productivas, organizativas, sociales, culturales y ambientales en su conjunto que actúan en una determinada zona geográfica, considerada como espacio turístico.

Una forma simple de pensar en el tema es a través de la suma de varios órganos sociales existentes en cada dimensión, así como sus acciones y expresiones. En general, tratase aquí de los diferentes sectores de la sociedad, por ejemplo, el sector primario, secundario y terciario. Otra posibilidad es agrupar estas organizaciones por su finalidad, u organizaciones privadas (con fines de lucro en general), las organizaciones estatales (locales, estatales y federales), las organizaciones públicas y no gubernamentales (ONG, asociaciones civiles, vecinales, etc.) 
En el segundo nivel, el micro o de las organizaciones (que son las unidades de producción), la recolección y el análisis de datos ocurre dentro de cada entidad colectiva que se mencionó anteriormente. Significa que al aplicar un "zoom" y cambiar el foco de análisis, se verá lo que sucede dentro de los órganos sociales (empresas, asociaciones, ONG, etc.), para ver cómo actúan. Para ello, algunas preguntas son necesarias y fundamentales para entender el funcionamiento de estas organizaciones y, en general, el funcionamiento de la actividad turística en el territorio. Por ejemplo: ¿cuáles son las prácticas de gestión del medio ambiente (dimensión ambiental) que se efectúan?; ¿las empresas consiguen ganancias que les permitan reinvertir en el territorio, aumentando, por ejemplo, sus negocios y la creación de puestos de trabajo (dimensión económica)?; ¿ $\mathrm{Si}$ las relaciones laborales se formalizarán en las empresas, por consecuente los derechos y condiciones de trabajo serán dignas (dimensión social)?; y ¿Existe transparencia en la información y/o participación de los trabajadores en las decisiones (dimensión ética)?, entre otras.

Además de estas dos escalas verticales de análisis, el método ODIT propone en la fase Retrato del Lugar. En este se puede observar la realidad compuesta por la dimensión ambiental, económica, social y ética, mediante el análisis, horizontal y dimensional, que en resumen generará el panorama turístico del destino investigado. Para cada uno de ellos hay también un conjunto de indicadores o elementos de medición para el control, como turistas o prácticas socioeconómicas en general que se están desarrollando en el territorio. (Cuadro 1)

Los indicadores ${ }^{2}$ son en todas las ciencias, "instrumentos de información" para apoyar a la gestión. Un indicativo de la importancia y la prioridad de una zona turística, en términos de acciones, inversiones y difusión, es la disponibilidad de información turística. A pesar de la creciente valoración y repercusión del turismo en la sociedad, las medidas de creación y utilización de indicadores turísticos aún son incipientes, quedándose muchas veces (cuando hay) restringidas a grandes ciudades y a los centros turísticos ya establecidos, sea en función de su atracción física, escénicas o paisajísticas; por sus centros económicos y/o políticos; por su relevancia histórico-culturales.

El uso de indicadores que potencien la capacidad de transmitir información, agregan valor a ella y permiten profundizar los análisis por medio de la correlación entre informaciones. El tratamiento diferencial de los datos puede generar información más objetiva y accesible a sus

CUADRO 1

Criterios para el abordaje en las escalas del territorio y de la empresa.

\begin{tabular}{lllll}
\multicolumn{1}{c}{ Dimensiones } & \multicolumn{1}{c}{ Ambiental } & \multicolumn{1}{c}{ Económica } & \multicolumn{1}{c}{ Social } & Ética \\
Critérios & Renovación & Rentabilidad & Reconocimiento & Gobernabilidad \\
& Prevención & Integración Territorial & Inserción & Transparencia \\
& Valorización & Flexibilidad & Práctica de Asociación & Solidaridad \\
& Cuadro de Vida & Perennidad & Accesibilidad & Tolerancia \\
\hline
\end{tabular}

Fuente: adaptado de AFIT (2001).

2. Los indicadores son datos numéricos relativos al menos a dos variables y tienen una unidad de medida y de referencia / comparación (OCDE, 1994). Como herramientas de apoyo a las decisiones, los indicadores han simplificado los modelos de la realidad con la capacidad para facilitar la comprensión de los fenómenos, la capacidad de comunicar los datos en bruto y la adaptación de la información a la lengua de los tomadores de decisiones. Las distintas categorías de indicadores se han utilizado en todo el mundo para diferentes propósitos: ambientales, sociales, culturales, históricos, turísticos, entre otros. 
usuarios, facilitando la comunicación entre la comunidad técnica y científica y la sociedad. A modo de ejemplo, se puede citar la capacidad de indicadores ambientales para retratar el potencial del turismo espacial y la capacidad de respuesta de los indicadores para monitorear las acciones e iniciativas de la gestión del turismo.

Es precisamente en este sentido que se inserta la tabla de indicadores en el método ODIT, la cual cumple doble función: ser una herramienta rigurosa para la recopilación de datos y la investigación científica y también proporcionar bases confiables para su control y la gestión del turismo.

De este modo, a través de un conjunto de indicadores para cada dimensión, se puede recopilar información que será analizada y retratada como los impactos del turismo. A su vez, la suma de los retratos de las cuatro dimensiones del lugar (económica, social, ambiental y ética) puede evaluar la magnitud de las empresas turísticas y la escala del territorio, y así obtener una evaluación global del turismo en cualquier destino.

Cada indicador proporciona información específica. Como ejemplo de indicadores turísticos se puede crear un indicador sobre el "impacto económico del turismo en la localidad" con el fin de saber cuánta inversión financiera entra en la localidad en función del turismo y estimar la proporción en que este afecta a otras actividades. Esto requeriría la obtención de datos sobre el volumen de los ingresos procedentes del turismo y cruzarlos con la cantidad de empresas en el sector primario (agricultura), secundario (industria) y terciario (servicios, con exclusión de su giro) que proporcionan productos para el turismo en un período determinado (por ejemplo, un año) (Cuadro 2).

Se debe considerar que antes de hacer un indicador es necesario saber quiénes son los proveedores de materias primas y productos para el sector turístico en el municipio. Esta es la información que debe recogerse en el comienzo de la fase 1 (Retrato del Lugar), en la que se identifica quiénes son las partes interesadas en las escalas de las empresas y del territorio.

En resumen, hay que destacar que el Retrato del Lugar no es lineal y requiere constantemente una revisión de los datos de campo, observar, clasificar, medir para obtener el mejor resultado posible. Por lo tanto, es esencial analizar el territorio, su diversidad, lo que puede ofrecer al turismo; además de observar cómo interactúan los diferentes sectores de la ciudad con el turismo y buscar maneras de beneficiarse mutuamente para nivelar las cuestiones del turismo en el territorio.

\section{Fase 2: El diagnóstico}

Su objetivo es interpretar en términos de desarrollo, los signos y las direcciones observadas en el Retrato del Lugar. Las señales pueden traducirse como amenazas, oportunidades, fortalezas y

\section{CUADRO 2}

Ejemplos de Indicadores de la Dimensión Ambiental en la Escala del Territorio.

Cuestión de dirección: ¿el turismo permite la renovación de los recursos ambientales sobre los cuales se apoya? ¿Eses recursos están amenazados por otras actividades realizadas en el territorio?

\begin{tabular}{ll}
\hline \multicolumn{1}{c}{ Indicadores posibles } & \multicolumn{1}{c}{ Elementos de mensuración $\left(\mathrm{n}^{\circ}\right.$ absoluto o porcentaje) } \\
Atracción paisajística y biodiversidad & Tasa de cambio de las características paisajísticas \\
Consumo del agua & Número de días con cuantidad de agua limitada \\
Cualidad del aire & Número de días con picos de polución \\
Cualidades sonora y olfativa & Número de empresas pulidoras por zona geográfica \\
Cualidad arquitectónica & Estado de patrimonio antiguo y moderno \\
Intensidad turística & Densidad de visitantes en los espacios $\left(\mathrm{m}^{2} \circ \mathrm{Km}^{2}\right)$ \\
\hline
\end{tabular}

Fuente: adaptado de AFIT (2001). 
debilidades, de acuerdo con los diferentes tipos de actores. Se debe definir la sostenibilidad para interpretar las implicaciones que efectúan las prácticas turísticas. El diagnóstico se centra en el turismo y en su integración con otros tipos de actividades y sectores, el cual está estrechamente involucrado con:

- los problemas originalmente identificados, es decir, hilo conductor de todo el análisis;

- la calidad de los métodos utilizados para el Retrato del Lugar, y su capacidad de considerar la diversidad de actores y sus representaciones;

- la capacidad de enumerar las "conclusiones" para el diagnóstico de los elementos observados en el Retrato del Lugar;

- el control del volumen de información recopilada para generar indicadores de turismo sostenible;

- la forma de organizarse colectivamente para construir y validar las interpretaciones (calidad de animación y la diversidad de la representación).

La experiencia evidencia que las prácticas no son dicotómicas, es decir, exclusivamente sostenibles o insostenibles. Ellas nunca se manifiestan en el todo de una misma manera, pero sí en grados de preponderancia. Hacer un diagnóstico, por lo tanto, no tiene la ambición de realizar un estado del arte del lugar, sino observar los actores involucrados y sus acciones que interfieren en el desarrollo sostenible.

Así pues, la etapa del diagnóstico trata de:

- identificar y validar colectivamente los temas de prioridad para su interpretación. Tratar de validar si la sostenibilidad de las prácticas turísticas responde a cuestiones prioritarias del territorio;

- fomentar el desarrollo de interpretaciones contradictorias (en el territorio las declaraciones y opiniones son contradictorias). Esta discusión implica la argumentación e induce al debate, basado en el marco de indicadores, con el objetivo de su rectificación y validación colectiva. El objetivo es tomar una dinámica que genere un diagnóstico turístico mínimamente compartido y consensuado;

- discutir y debatir colectivamente para completar el diagnóstico del territorio turístico. Plantearse una base de indicadores claros y comunes; problemas, prácticas y representaciones del trabajo y sobre la diversidad de puntos de vista (interpretaciones contradictorias) que posibilite validar colectivamente el diagnóstico del territorio.

Como resultado de esta etapa se elabora una lista de sugerencias sobre los aspectos que deben ser mejorados en el territorio y los negocios.

\section{Fase 3: Elaboración de ejes estratégicos}

A partir del diagnóstico validado por todos los actores involucrados, en esta fase se definen las principales líneas de trabajo para comparar las fortalezas y mejorar las debilidades del turismo. La idea es diseñar acciones que tengan algún grado de estabilidad en el tiempo. En general se utiliza un marco de tiempo de 3 hasta 5 años como referencia para concebir un plan de acción.

Ahora, se deben definir y priorizar colectivamente las áreas de intervención estratégica en el territorio. Los ejes son los objetivos de desarrollo de los intermediarios del lugar (por ejemplo, mejorar la calidad del agua, mejorar la rentabilidad del negocio, entre otros) y se extraen de los puntos identificados en el diagnóstico para ser mejorados.

Para validar los ejes estratégicos a debatir conjuntamente por el grupo local, se necesita:

- analizar los vínculos entre la sostenibilidad y tipos de prácticas turísticas llevadas a cabo en el territorio y su impacto en la región;

- proponer ejes estratégicos teniendo en cuenta el conjunto de elementos validados en el diagnóstico;

- organizar el debate para establecer prioridades y colectivamente validar los ejes estratégicos que deben conservarse para el territorio (la dimensión temporal es uno de los elementos que requiere reflexión). 
La organización propuesta, vinculada al método de investigación, facilita la síntesis, porque en esta etapa los actores tienen dos tipos comunes de referencias: una identificar los tipos de turismo y su práctica con la sostenibilidad, y otra evaluar el impacto de estas prácticas en el desarrollo del territorio. El conjunto es apreciado a partir de una herramienta común: la tabla de indicadores de turismo local.

Para establecer la jerarquía de los objetivos intermedios (también llamados líneas de acción) debe tomarse en cuenta la cuestión: ¿las directrices propuestas permiten atender (corregir/mitigar) las observaciones inicialmente realizadas? De esta forma, dicha etapa permite relacionar la coherencia de la trilogía del procedimiento: búsqueda / interpretación / metas prioritarias del desarrollo.

\section{Fase 4: El plan de acción}

Esta fase consiste en organizar y priorizar en el tiempo y en el espacio las acciones correspondientes a los principales objetivos de desarrollo identificados anteriormente (en la etapa de los ejes estratégicos). La cuestión central en el plan de acción es: ¿las acciones propuestas cumplen el objetivo del desarrollo orientado correctamente (prioridades estratégicas)?

Para aclarar esta cuestión (elaboración del plan de acción y validación de forma colectiva) es útil volver a lo que ya se hizo. Por lo tanto, es preciso volver a las constataciones realizadas en el Retrato del Lugar, el cual está lleno de enseñanzas para orientar el debate de las acciones propuestas adaptadas.

Se recomienda tener una discusión sobre los hechos (por ejemplo, la mejora de la calidad del agua - Eje Estratégico 1 - puede no ser percibida como una prioridad de todos los interesados. Cada uno puede tener su visión sobre el tema lo que requerirá diferentes acciones para tratar del problema. Por ejemplo, para ciertos actores puede ser un problema de conciencia, para otros será un problema económico).
La pregunta que surge aquí es si se puede actuar simultáneamente sobre una serie de instrumentos existentes o sólo unos pocos (uno a la vez) Para aclarar tal duda es necesario un análisis sistémico comprometido, así los actores deben desarrollar sistemáticamente el plan para lograr el medio, el elemento humano y la logística necesaria para poner en práctica cada acción de inversión designada.

\section{Seguimiento y evaluación: \\ un proceso transversal}

El seguimiento y la evaluación se realizan en dos ocasiones: al final de cada etapa y con una guía para la toma de decisiones. En caso de tener el marco de indicadores, se debe buscar la información de registro a lo largo de cada paso, para revelar las razones y elegir un camino y al mismo tiempo construir una base de datos temporal. Se puede hacer directamente con los actores del marco de indicadores. La segunda es al final del ciclo de las cuatro etapas donde se termina con la elaboración del plan. Lo mismo puede ocurrir cuando se lo pone en práctica, con evaluaciones a cada etapa y también al final de cada ciclo.

\section{Conclusión}

Los estudios sobre planificación del turismo pasarán por un largo período de estancamiento, especialmente cuando se trata de teorizar y ampliar el conocimiento sobre el proceso de planificación per se. El método ODIT se diferencia de otros modelos de planificación del turismo al guiarse por la búsqueda de la interrelación entre todos los actores que participan en la industria del turismo y la flexibilidad para crear indicadores alineados a la realidad de cada destino. Otros modelos de planificación restringen la participación de las partes interesadas en el proceso (planificación centralizada). Por otra parte, las metodologías de planificación interactiva, a menudo, son más exitosas en contextos individuales, 
por lo tanto, no son absolutamente reproducibles en otros contextos.

En el caso de la ODIT, se fundamenta por criterios de flexibilidad para la creación de marcadores y promueve la adaptación del modelo para diferentes contextos. Además, la ODIT excede las acciones de la cartografía y la elaboración de planes rígidos, que proporcionan herramientas de análisis para el control y la evaluación de la necesidad de medidas de adaptación, en forma de indicadores de turismo, lo que puede resultar en la toma de decisiones y la aplicación de plan calificado.

Por lo tanto, el mérito de la metodología ODIT representa un avance indiscutible, pero también presenta algunas limitaciones, en que hace falta un progreso, tales como la creación de nuevas dimensiones e indicadores para el análisis. También se recomienda la revisión de su lógica de funcionamiento para especificar las acciones y profundizar la precisión del proceso. La profundización de la investigación empírica en el contexto de Brasil puede probar y extender la metodología que conduce a un mejor margen para iniciativas estratégicas y lograr resultados beneficiosos en el desarrollo de la visita planeada.

\section{Referencias Bibliográficas}

AFIT - Agence Française de l'Ingénierie Touristique (2001) Piloter le Tourisme Durable dans les Territoires et les Enterprises: guide de savoir-faire. Paris.

Acerenza. M. A. (2003) Administração do Turismo. Bauru: EDUSC.

Aglietta, M. (1976) Régulation et crises du capitalisme. Calmann-Levy. Paris.

Boyer, M. (2003) História do turismo de massa. São Paulo: Editora EDUSC.

Boyer, M. \& Saillard, Y. (Eds.) (1995) Théorie de la régulation. L'etat des savoirs. La Découverte, Paris.

Boyer, R. (1986) La théorie de la régulation: une analyse critique, La Découverte, Paris.

Costa, C. (2001) "An emerging tourism planning paradigm? A comparative analysis between town and tourism planning". International Journal of Tourism Research, $\mathrm{n}^{\circ} 3,425-441$.

EMBRATUR - Instituto Brasileiro de Turismo (2007) "Elaboração do Plano Estratégico de Desenvolvimento do Turismo Regional'. (Módulo Operacional nº4). Brasília (DF): MINTUR.

Fayol, H. (1931) Administration industrielle et generale: prevoyance, organisation, commandement, coordination; controle. Paris: Dunod. 174p.

Harvey, D. (1996) Condição Pós-Moderna: uma pesquisa sobre as origens da mudança cultural. 6 Ed. São Paulo: Loyola.

IFEN - Institut Français de l'Environnement (1999) Les Indicateurs de Développement Durable - Méthodes et Perspectives. Orléans: Corbet, Études et Travaux. 145 p.

Kuhn, T. (1962) The structure of scientific revolutions, University of Chicago, 172p., Chicago.

Lipietz, A. (1977) Le capital et son espace. Paris: La Découverte.

Lohman, G. \& Panosso Netto, A. (2008) Teoria do turismo. Conceitos, modelos e sistemas. São Paulo: Editora Aleph, (Série Turismo). 468p.

OECD - Organization for Economic Cooperation and Development (1994) Environmental Indicators. Paris: OECD. pp. 124-176.

Pimentel, T. D. (Org). (julho, 2010) Plano Estratégico para o Desenvolvimento Sustentável do Turismo no município de Lima Duarte/MG. Relatório Final da Disciplina de Planejamento e Organização do Turismo II. Versão não publicada. Universidade Federal de Juiz de Fora.

Pimentel, T. D. (Org). (julho, 2011) Plano Estratégico para o Desenvolvimento Sustentável do Turismo no município de Caxambu/MG. Relatório Final da Disciplina de Planejamento e Organização do Turismo II. Versão não publicada. Universidade Federal de Juiz de Fora.

Pimentel, T. D. (Org). (julho, 2012) Plano Estratégico para o Desenvolvimento Sustentável do Turismo no municipio de São Lourenço/MG. Relatório Final da Disciplina Gestão de Destinos Turísticos. Versão não publicada. Universidade Federal de Juiz de Fora.

Pimentel, T. D. (Org). (julho, 2013) Plano Estratégico para o Desenvolvimento Sustentável do Turismo no município de Juiz de ForalMG. Relatório Final da Disciplina Gestão de Destinos Turísticos. Versão não publicada. Universidade Federal de Juiz de Fora.

Pimentel, T. D. \& Carvalho, Fabíola C. C. (2014) "Fatores Condicionantes do Planejamento e Gestão em Destinos 
Turísticos: um quadro teórico de análise”. TURyDES, Málaga, vol. 1, p. 1-37.

Pimentel, T. D. \& Carvalho, Fabíola C. C. (2013) "Mapeando os Modelos de Planejamento Turístico: em busca de refinamento teórico com vistas à intervenção qualificada". In: Anais do X Seminário da Associação Nacional de Pesquisa e Pós-Graduação em Turismo / ANPTUR, 2013, Caxias do Sul (RS). Caxias do Sul (RS): Editora da Universidade de Caxias do Sul / EDUCS, 2013. vol. 1. p. 1-15.

Pimentel, T. D.; Emmendoerfer, Magnus L. \& Tomazzonni, Edegar L. (Org.). (2014) "Gestão Pública do Turismo no Brasil: teorias, metodologias e aplicações”. (1. Ed.) vol. 1. Caxias do Sul (RS): Editora da Universidade de Caxias do Sul / EDUCS. 528p.

Stiftel, B. (2000) Planning Theory: The National AICP Examination Preparation Course Guidebook. Ed. Roshi Pelaseyed \& Am. Inst. Cert. Planners: Washington DC. pp.4-16. Recuperado de: <garnet.acns.fsu.edu/ bstiftel/ STIFTEL_AICP_Planning_Theory_Chapter.pdf $>$. Aceso: 01 jan 2010.

Vandenberghe, F. \& Boschi, R. (Org.) (2013) Estudos Exemplares (Manuscrito). Curso para Pós-Graduação (M/D). Estudos Sociais e Políticos/IESP. Universidade do Estado do Rio de Janeiro/UERJ. 
